\title{
3-Dimensional Photogrammetric Measurement of Magnetic Field Lines in the WEGA
}

\author{
Stellarator \\ Peter Drewelow, Torsten Bräuer, Matthias Otte, Friedrich Wagner, Andreas Werner \\ Max-Planck-Institut für Plasmaphysik, EURATOM Association, \\ Wendelsteinstr. 1, D-17491 Greifswald, Germany*
}

\begin{abstract}
The magnetic confinement of plasmas in fusion-experiments can significantly degrade due to perturbations of the magnetic field. A precise analysis of the magnetic field in a stellarator type experiment utilizes electrons as test particles following the magnetic field line. The usual fluorescent detector for this electron beam limits the provided information to two-dimensional cut views at certain toroidal positions. However, the technique described in this article allows measuring the 3-dimensional structure of the magnetic field by means of close-range photogrammetry. After testing and optimizing the main diagnostic components, measurements of the magnetic field lines were accomplished with a spatial resolution of $5 \mathrm{~mm}$. The results agree with numeric calculations, qualifying this technique as an additional tool to investigate magnetic field configurations in a stellarator. For a possible future application ways are indicated, how to reduce experimental error sources.
\end{abstract}

PACS numbers: 07.68., 41.75., 52.20., 52.20., 52.55.

\section{INTRODUCTION}

The two main concepts for confining high temperature fusion plasmas by magnetic fields are tokamaks and stellarators. Both concepts are based on toroidal systems with helically twisted magnetic field lines. A measure of the twist of the magnetic field lines is the rotational transform $t$, which is defined as the ratio between poloidal and toroidal angular displacement of the field line. In case of this ratio being not rational, the magnetic field lines form nested magnetic flux surfaces as shown in figure 1 a). Lying on these flux surfaces, the magnetic field lines can be

*Electronic address: peter.drewelow@ipp.mpg.de 
a)

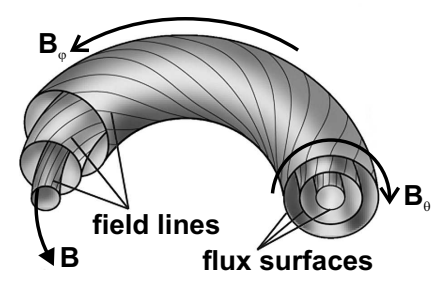

b)

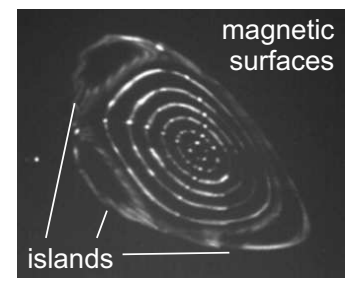

Figure 1: (a) Twisted toroidaly closed magnetic field lines form nested flux surfaces. (b) Experimentally determined Poincaré plot showing a poloidal cut-view of multiple nested flux surfaces with three islands in the outer region of the WEGA stellarator ${ }^{1}$.

described by a poloidal $B_{\theta}$ and toroidal $B_{\varphi}$ magnetic field component. In tokamaks the helical twist of the field lines is generated by a strong plasma current. In stellarators both magnetic field components are mainly provided by magnetic field coils outside of the plasmas.

At certain low order rational values of the rotational transform, resonant field error structures can occur which appear in the form of so called magnetic islands. These are smaller, closed and off-center magnetic surface structures, similar to the nested magnetic surfaces in the central region. Figure $1 \mathrm{~b}$ ) shows a cross sectional view of the nested magnetic surfaces in the WEGA stellarator with three islands at $t_{a}=1 / 3$ in the outer region. In general, islands are either induced by the periodicity of the magnetic field of the stellarator or by additional perturbing magnetic fields. The existence of both field error structures was shown in different stellarator experiments (see e.g. ${ }^{1,3-6}$ ). Parallel to the magnetic field lines, particles can freely propagate and transport energy. Crossing the x-points of these islands, the particle flow contributes to an increased radial transport as well. Thus, a decrease of the confined plasma energy can be observed in these perturbed magnetic configurations ${ }^{2}$. To understand the confinement of a stellarator, it is therefore necessary to characterize the magnetic configuration and examine closed and nested flux surfaces with regard to possible field perturbations.

Since the vacuum field in a stellarator - produced by external coils only - is already confining, this check of field integrity can be performed in the first step without plasma. A common method ${ }^{1,3-6}$ to survey the field structure utilizes electrons as test particles. Due to the Lorentz force, these electrons gyrate perpendicular to the field lines, while the parallel particle motion is essentially unimpeded. Free electrons can be generated and accelerated to a defined initial energy by an electron gun. To detect the emitted electron beam in a poloidal cross section, a fluorescent mesh or a moving fluorescent bar is commonly used ${ }^{1,3-6}$. The luminous intersection points of the beam can be photographed 
and present a cut view through the magnetic flux surfaces (Poincaré plot). Figure 1 b) shows such a Poincaré plot for different radial positions of the electron beam from the magnetic axis to the last closed flux surface, merged into one picture for the WEGA stellarator ${ }^{1}$.

This common method can only provide two-dimensional cross-sectional views of the three-dimensional magnetic field structure, although the electron beam trajectory follows the whole field line on its flux surface. In order to gain spatial information on the magnetic field configuration, it is possible to use a background gas (e.g. argon) as an alternative detector for the electron beam. As shown before ${ }^{8}$, an electron beam in a background gas creates a luminescent trace following the beam path, due to electron-impact excitation of the gas particles and subsequent emission of light. The trace is visible in the whole stellarator and can be observed from different viewpoints. To measure the spatial structure of such a luminescent trace, pictures from different viewpoints are analyzed by means of photogrammetry. Further information on the photogrammetry setup are provided in section IV.

The field line survey has been performed at the WEGA ${ }^{11}$ stellarator operated at Max-Planck-Institut für Plasmaphysik, Greifswald. WEGA is a classical 5-field period stellarator with a major radius of the plasma vessel of $R_{0}=72 \mathrm{~cm}$ and a minor radius of $r_{0}=19 \mathrm{~cm}$, respectively. A magnetic field strength of $B_{0}=50 \ldots 600 \mathrm{mT}$ for typically flat-top times of $t=30 \mathrm{~s}$ can be generated by forty toroidal magnetic field coils. Two pairs of helical field coils $(l=2$ and $m=5)$ induce the twist of the field lines, with a normalized rotational transform of $t=\iota / 2 \pi$ ranging from 0.1 to 1 , depending on the ratio of the currents in the helical and poloidal coils. The maximum plasma radius is $a \approx 11 \mathrm{~cm}$. The plasma vessel can be evacuated to UHV conditions $\left(p \leq 1 \times 10^{-7} \mathrm{mbar}\right)$ and a gas inlet system can provide argon or other gases at the required pressure.

The technique described in this paper demonstrates for the first time a three-dimensional measurement of the magnetic field lines in a toroidal section of the stellarator. In addition to the detailed cut views of the fluorescent-bar method, it provides spatial information on field configurations.

\section{PRECEDING CALCULATIONS}

Regarding the interpretation of the measurement data, a clear discrimination between the luminescent trace, the drift path of the electrons and the magnetic field line is indispensable. The drift orbit of the electrons does not coincide 
with the magnetic field line because of two effects. First, the gyration produces a deviation of one gyro-radius ${ }^{7}$

$$
r_{G}=\frac{|\vec{p}|}{e_{0} B} \cdot \sin \alpha
$$

with $\vec{p}$ the momentum and $e_{0}$ the charge of the electron, $B$ the strength of the magnetic field $\vec{B}$ and $\alpha$ the pitch angle between $\vec{p}$ and $\vec{B}$. However, for the experiments at the WEGA stellarator, discussed in the following sections, this deviation is limited to $r_{G} \leq 0.1 \mathrm{~mm}$. Second, a drift velocity $v_{D}$ is generated by the curvature and gradient of the magnetic field ${ }^{7}$

$$
v_{D} \approx \frac{m_{e}}{e_{0}}\left(v_{\|}^{2}+\frac{1}{2} v_{\perp}^{2}\right) \frac{\vec{B} \times \overrightarrow{\nabla B}}{B^{3}}
$$

with the electron velocity components $v_{\|}$and $v_{\perp}$ parallel and perpendicular to $\vec{B}$, respectively. It displaces the electron drift path vertically from the field line, while the particle is orbiting in the upper (or lower) half of the stellarator. During the same orbiting time $t_{O}$ in the respective other half, the drift path returns to the field line. To estimate the maximum vertical displacement from the field line, a constant drift $r_{D}=v_{D} \cdot t_{O}$ can be assumed. In WEGA, with the field strength of $B=0.6 \mathrm{~T}$ and the velocities $v_{\|}$and $v_{\perp}$ of the electrons used in section IV, this displacement is up to $r_{D} \leq 1.8 \mathrm{~mm}$. The visible trace of excited gas particles exhibits an additionalbroadening with respect to the electron beam, because of thermal motion of the atoms and ions during the lifetime $\tau$ of an excited state. In argon the lifetime of optical transitions (wavelength $300 \mathrm{~nm} \leq \lambda \leq 800 \mathrm{~nm}$ ) with the maximum duration for the transition from $3 s^{2} 3 p^{5}\left({ }^{2} P^{\circ}{ }_{3 / 2}\right) 4 s$ to $3 s^{2} 3 p^{5}\left({ }^{2} P^{\circ}{ }_{3 / 2}\right) 5 p(\lambda=420 \mathrm{~nm})$ is $\tau_{\max }=1 \mathrm{~ms}^{9}$. Assuming argon to be at room temperature, the luminescent trace will be additionally broadened by up to $\Delta r_{e x c} \leq 0.45 \mathrm{~mm}$. Thus, the drift path of the electrons is accepted as a good approximation of the magnetic field line with an inaccuracy in the order of a few millimeters.

\section{OPTIMIZATION OF MEASUREMENT COMPONENTS}

Prior to the application of the magnetic field line measurement technique at the WEGA stellarator, the components (electron gun, background gas, magnetic field) were surveyed and optimized with respect to the following objectives:

- a high luminescence and good contrast of the transitions of the visible trace in the images,

- a small diameter across the electron beam and a low divergence of the beam and

- a long visible range of the luminescent trace. 
a)

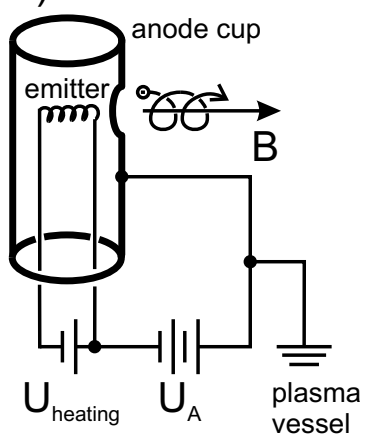

b)

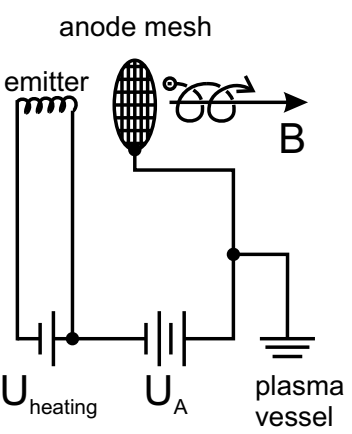

c)

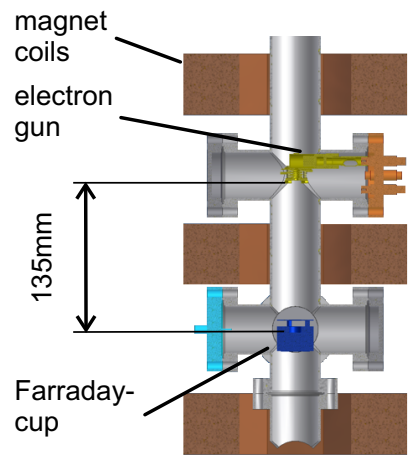

Figure 2: (Color online) Two designs for an electron gun are shown, accelerating the electrons from a heated and negatively charged tungsten filament with a metal-cup with a hole in flight direction (a) and with a mesh in flight direction (b). The anodes are on the same potential as the plasma vessel to screen electric fields. (c) Cut view of the vacuum chamber with magnetic field in axial (vertical) direction where preliminary tests of the electron guns were performed.

The emission of light, as well as the divergence of the beam is caused by electron collisions with gas particles. Therefore, the main influences on the electron beam and the atomic scattering processes were studied in detail. The accessible experiment parameters are the gas pressure, the magnetic field strength as well as the design and operation parameters of the electron gun. For easy access and adaptability the first investigations on the electron beam characteristics were conducted at a linear vacuum chamber with magnetic field as shown in figure $2 \mathrm{c}$ ).

The requirement for a high contrast of the visualized field lines in the images can be met by different means. To increase the excitation rate of the gas particles, a high current in the electron beam is necessary. Different designs of an electron gun were studied and characterized by their emission properties to reach high emission currents. Figure 2 shows two promising designs which were tested. In both cases free electrons are generated through thermal emission from a tungsten wire and accelerated in an electric field. Due to the tying effect of the magnetic field, focusing 


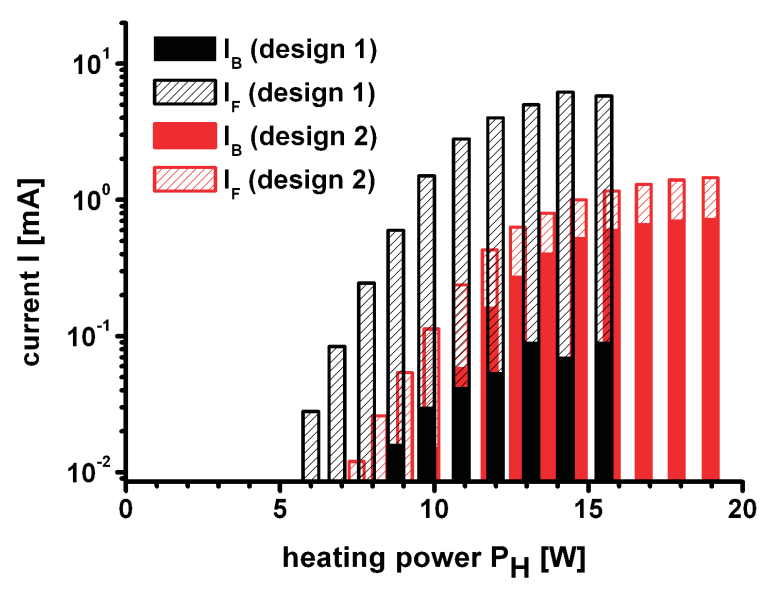

Figure 3: (Color online) Electron current emitted from the tungsten filament $I_{F}$ (shaded bar) and current in the electron beam $I_{B}$ (full bar) for different heating powers.

electrodes are not needed and would rather disturb the beam because of unintended $\vec{E} \times \vec{B}$ drifts. The electrons are accelerated to a defined initial energy $E_{0}=e_{0} U_{A}$ by the acceleration voltage $U_{A}$ applied in design 1 (fig. 2 a) to a metal cup with a hole and in design 2 (fig. 2 b) to a metal mesh.

The emitted beam currents of both designs were measured with a Faraday cup after a free flight distance of $\Delta s \approx 135 \mathrm{~mm}$. The heating power $P_{H}$ serves as a quantity to describe the temperature $T_{F}$ of the filament, which, was not measured. The higher $T_{F}$, the more electrons can overcome the work function and leave the metal surface. As can be seen in figure 3 , the current $I_{F}$ emitted by the filament (shaded bars) of design 1 (black) is higher than $I_{F}$ of design 2 (red). However, design 2 transfers it with a higher efficiency in the beam current $I_{B}$ (full bars). Electrons, which drift along the magnetic field can exit the metal cup through the hole (to the right in figure 2 a) or move in the opposite direction and hit the cup (to the left in figure 2 a). Thus a higher current loss in contrast to design 2 (red bars) is caused. The saturation of $I_{F}$ for higher heating powers is expected to be due to charge accumulation in the vicinity of the filament. The saturation current $I_{F, \max }$ is proportional to $U_{A}^{3 / 2}$ according to the Child-Langmuir law ${ }^{10}$. To avoid arcing inside the gun between filament and anode, the acceleration voltage was limited to $U_{A}=300 \mathrm{~V}$.

The structure and placement of the anode determines not only the beam current $I_{B}$ but also the initial average pitch-angle $\alpha$ between electron momentum $\vec{p}$ and magnetic field vector $\vec{B}$. According to equation (1) this has an influence on the mean radius of gyration of the electrons $r(\alpha)$. In every scattering event where electrons change their 
a)

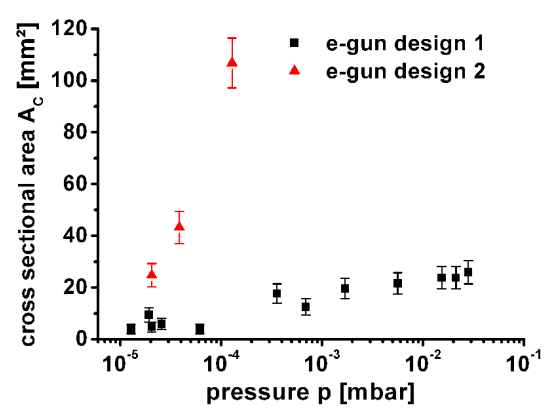

b)

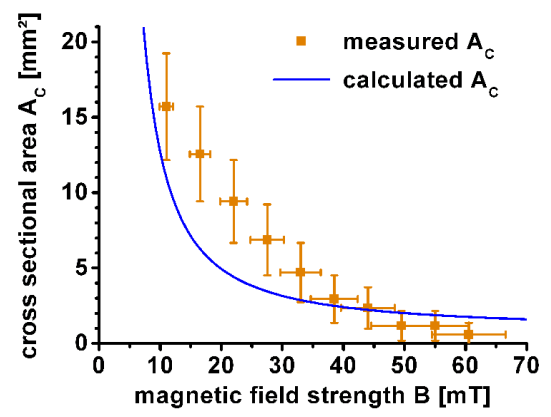

Figure 4: (Color online) (a) Measured cross sectional area $A_{C}$ for different pressures $p$ of argon at $B=22 \mathrm{mT}$ after a flight distance of $\Delta s=135 \mathrm{~mm}$ shown for two electron gun designs operated at $U_{A}=40 \mathrm{~V}$. (b) Calculated and measured $A_{C}$ for different magnetic field strengths $B$ with electron gun design 1 .

momentum vector, they start rotating around a new gyro center which can be up to two gyro-radii away. Thus the divergence of the beam is not only dependent on the number of collisions but also on the pitch angle distribution among the electrons. Naturally an increased number of collision partners multiplies the scattering rate leading to a pressure dependence of the divergence. The Faraday cup in the setup (figure 2 c) was replaced with a fluorescent grid, which had a gauged lattice spacing of $1 \mathrm{~mm}$. Thus, it was possible to measure the diameter of the electron beam in two dimensions after the distance $\Delta s=135 \mathrm{~mm}$ from the electron gun for different gas pressures. An elliptic cross section could be observed and the area $A_{C}$ was calculated. The initial cross section was circular, determined by the shape of the tungsten filament (diameter $d_{F}$ ) projected in the direction of the magnetic field vector.

The cross sectional area can be estimated as $A_{C, 0}=\pi\left(1 / 2 d_{F}+r_{G}\right)^{2} \approx 4.4 \mathrm{~mm}^{2}$, taking also into account the projection of the gyro-motion of the electrons. Figure 4 (a) shows that the beam collimation is far worse in design 2 (red) and exhibits a stronger pressure-dependence compared to the beam of the electron gun in design 1 (black). 
Figure $4 \mathrm{~b}$ ) addresses the field dependence of the gyro radius (equation 1) and shows the measured cross sections with increasing field strength, highlighting, that a higher magnetic field strength prevents the beam to diverge rapidly. The calculated $A_{C}$ was derived from the above stated initial cross section taking into account the dependency on $r_{G}(B)$.

The length of the visible trace is limited due to the finite initial energy of the electrons $E_{0}$ and a continuous energy loss due to collisions. The rate coefficients for the inelastic scattering with atoms and ions are mainly dependent on the density $n_{\text {gas }}$ (respectively pressure $p_{\text {gas }}$ ) of the gas. Figure 5 b) and c) show pictures taken in the plasma vessel for different pressures and with the initial electron energy of $E_{0}=300 \mathrm{eV}$. From counting the visible transitions, the beam lengths in argon were estimated (figure 5 a) with an uncertainty of a half toroidal circumference (error bar). With higher pressures, the higher probability of inelastic scattering in a gas leads to a decreased range of the visible trace but increases its luminescence and hence the contrast in the images. However, for lower pressures, the multiple transitions interfere with each other, making an accurate counting of the visible traces difficult.

As necessary for the analysis, the brightness value of the visible trace has to exceed the background value by a certain selectable threshold. In case of the used analysis software ${ }^{12}$ this threshold was set to 30 intensity levels in the 8bit greyscale images (256 intensity levels). To get reliable results, the brightness gradient perpendicular to the light beam should be very steep. With respect to background light, design 2 has the disadvantage, that the emitted light from the tungsten filament illuminates the whole torus, further decreasing the contrast of the visualized field lines.

In addition, space charge effects in the beam originating from the negative electrons and positive argon ions, which could be detected in the luminescent trace, are supposed to have an influence on the propagation of the electrons. However, a thorough investigation of these effects would exceed the scope of this work.

As a result of the optimization, the following application of the method was performed with the electron gun design 1 (see fig. $2 \mathrm{a}$ ), operated at $P_{H}=10 \mathrm{~W}$ and $U_{A}=300 \mathrm{~V}$, emitting a current of $I_{B} \approx 5 \mathrm{~mA}$. The stellarator was filled with argon at a pressure of $p_{a r}=4 \times 10^{-4}$ mbar and a magnetic field strength of $B_{0} \approx 0.6 \mathrm{~T}$ and a rotational transform of $t \approx 1 / 3$ were applied. Thus, the beam created a visible trace of 5 revolutions, which is equivalent to a length of $s \approx 24 \mathrm{~m}$. The cross section diameter of the detectable beam increases up to $d \approx 5 \mathrm{~mm}$. The selected gas pressure, resulting in this rather short range (compare to figure 5 a), as well as the electron gun design 1 with the low emission efficiency (see figure 3) were chosen, because this proof of principle application focused on a high contrast 
a)

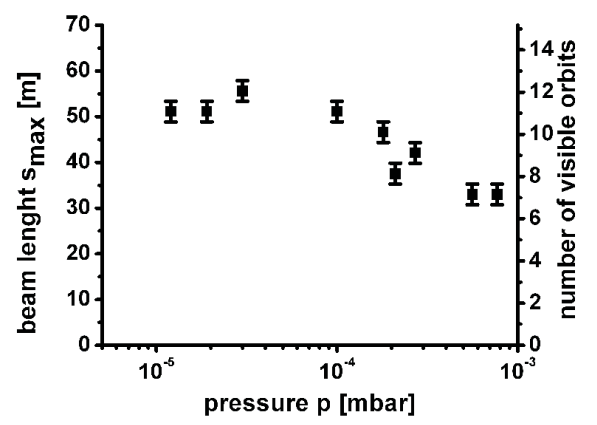

b)

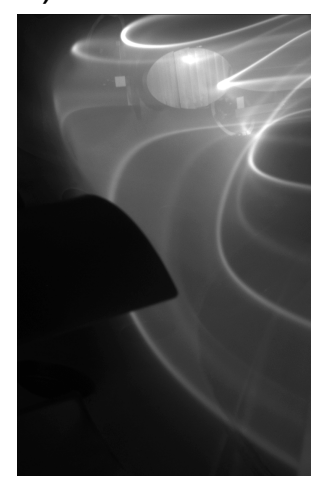

c)

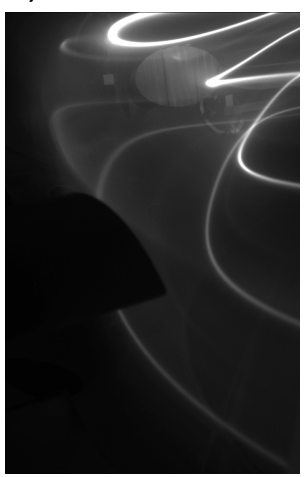

Figure 5: (a) length of the Visible beam $s_{\max }$ calculated from the number of visible orbits in argon vs. pressures $p_{A r}$ and tangential view on the visualized field lines for $p_{A r, b}=1.8 \times 10^{-4} \mathrm{mbar}(\mathrm{b})$ and $p_{A r, c}=7.7 \times 10^{-4}$ mbar (c) with electron gun design 1 .

and an easy analysis of the photos. Therefore, multiple trace transitions crossing each other in the field of view of the cameras as well as a bright background need to be avoided. For further applications of this technique with another focus on the beam characteristics, a different choice of electron gun and experiment parameters may be advisable.

\section{MEASUREMENT SETUP AND TECHNIQUE}

Figure 6 a) shows the measurement setup at the stellarator using four viewpoints with overlapping field of view. The pictures where taken by a Starlight Express HX916 TM camera using a Peltier cooled CCD-chip with a resolution of $1300 \times 1030$ pixel. In the common field of view of all cameras a set of six reference points was installed on the wall inside the plasma vessel. It served as a spatial reference and orientation for the analysis software and had to be visible from each point of view. To achieve this overlap, a lens with $f=8 \mathrm{~mm}$ focal length was mounted close to the vacuum 
a)

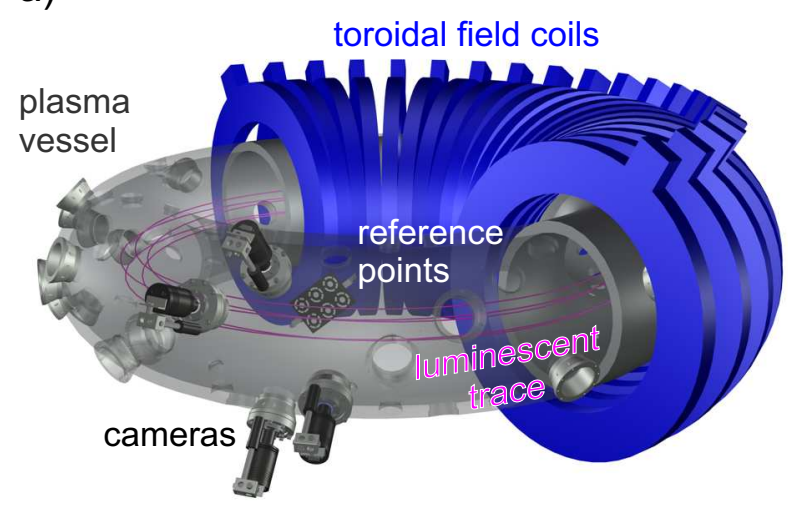

b)

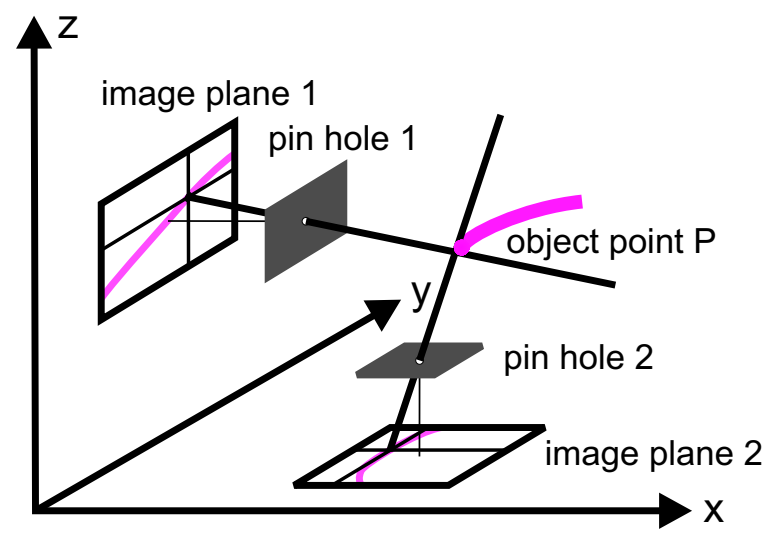

Figure 6: (Color online) (a) Device setup with toroidal field coils (dark blue), vacuum vessel (gray), luminescent trace of the electron beam (magenta), four observation cameras (black) and a mount with six reference points (white dots with rings). (b) Sketch of principle of photogrammetry with two pinhole cameras observing one object point.

vessel. As a consequence, this resulted in a non optimized depth of sharpness in the actual experimental setup.

To retrieve the 3-dimensional coordinates of the visible trace in the field of view of the cameras, the commercial photogrammetry software AICON 3D-Studio ${ }^{12}$ was applied. The principle of this photogrammetric technique is illustrated in figure $6 \mathrm{~b}$ ). First, the light ray connecting the image point (actually the pixel on the CCD-chip) with the object point $\mathrm{P}$ (actually the visualized field line), going through the pin hole (actually the lens system) was calculated. By taking two different pictures from the same object point (transition of the luminescent trace), the intersection point of the respective calculated light rays provide the measured coordinates. The precise optical and geometric properties of the lens system and image sensor had to be calibrated beforehand. The coordinates were scaled with respect to the distinct distance of two of the above mentioned six reference points, which were calibrated 


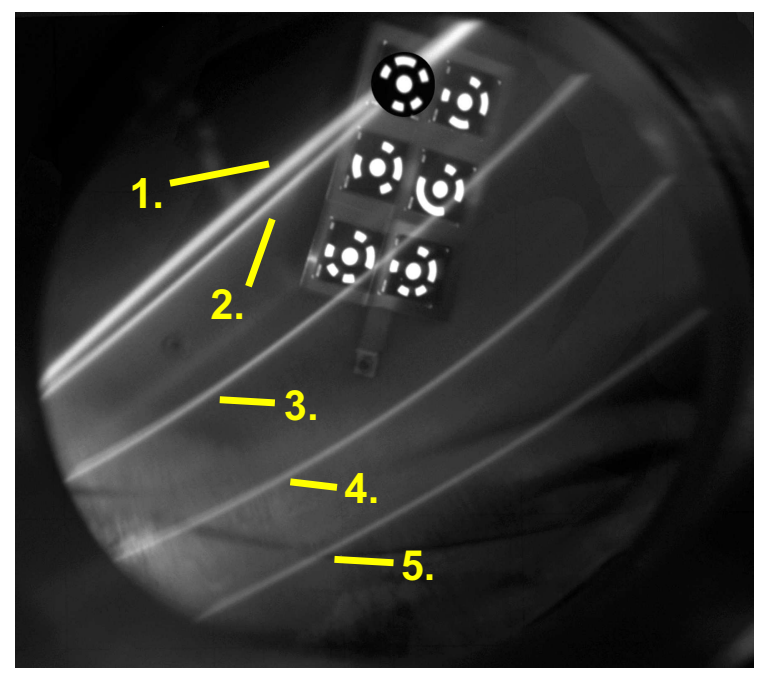

Figure 7: (Color online) Photo of one visualized magnetic field line with five transitions in the field of view and six reference points inside the torus.

outside the vessel with an accuracy of $\Delta r_{\text {scale }} / r_{\text {scale }}=0.02 \mathrm{~mm} / 103.15 \mathrm{~mm} \approx 2 \times 10^{-4}$. In addition, the reference points allow determining the location and orientation of the cameras in the WEGA coordinate system. Therefore, their coordinates in the stellarator coordinate system were calculated from the positions of the mounting points inside the vessel and the dimensions of the reference point carrier, measured with an accuracy of $\Delta r_{r e f}=0.7 \mathrm{~mm}$. It was not possible to verify the actual position of the mounted reference point carrier, because of the limited access to the mounting points inside the plasma vessel.

\section{DATA ANALYSIS AND RESULTS}

Photos of the luminescent traces and of the reference points were taken separately. As shown in figure 7 they were merged via photo editing software to handle the high contrast between reference points, traces and background. The composed pictures from the four viewpoints were processed by the photogrammetry software mentioned before.

The measurement procedure focused on the borders of the visible traces where the contrast was highest. To illustrate this, figure 8 shows two sample parts of the same field line section in two images. At first a border point (red point in fig. 8 a) in one picture was chosen and the software calculated the light ray going through the lens and striking the respective position on the image sensor. This ray was projected in the field of view of another camera (red line in fig.

$8 \mathrm{~b}$ ) and the respective border had to be indicated manually. The software then searched the contrast rich border in 
a)

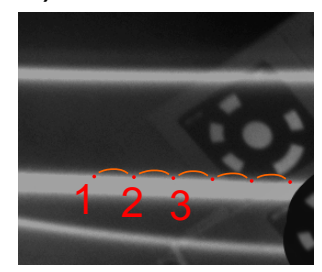

b)

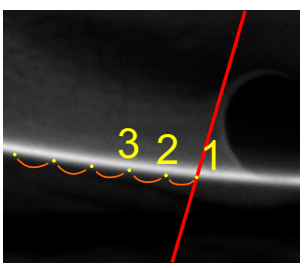

Figure 8: (Color online) Trace measurement principle shown with two images of the same field line section. Red and yellow points represent border points of the trace while the red line is the projection of a calculated light ray of point 1 of image (a) in the field of view of image (b).

the proximity of the first point and repeated those steps along the line till the end of the image or until the contrast was insufficient. Due to only partially sharp pictures of the traces (drawback of the demanded wide overlap of field of views), the automatic measurement succeeded only for short separated sections of only one border of the trace. A measurement of both borders of the light trace could have been used to calculate the average path between them, which would not be displaced from the actual electron trajectory by a half diameter of the luminescent trace.

It was possible to follow the beam trajectory and measure up to the $4^{\text {th }}$ revolution of one magnetic field line. The accuracy of the measured points is mainly influenced by

- the spatial resolution of the optical system $\Delta r_{O}=0.8 \mathrm{~mm}$,

- the inaccuracy of the photogrammetry process with the error of the coordinate transformation included $\Delta r_{P}=$ $0.8 \mathrm{~mm}$ and

- the deviation of the measured borders from the actual central core of the traces $\Delta r_{T}=3.3 \mathrm{~mm}$.

To demonstrate, to what extend this measurement of the $4^{\text {th }}$ revolution with a spatial resolution of $\pm 5 \mathrm{~mm}$ is sensitive to the rotational transform $t$, numerical calculations with modules of the W7 code were accomplished. This program is a field line tracing code calculating, among other things, drift paths of particles in the magnetic field of a given coil setup, with a guiding center approximation. It works similar to the GOURDON code ${ }^{13}$, but uses predictorcorrector methods instead of the Runge-Kutta algorithm. With this tool, the previously surveyed magnetic field configuration was calculated. In a second calculation, a configuration with a different rotational transform $t$ was studied. Figure 9 shows the calculated intersection points of an electron drift path in one poloidal plane (Poincaré 


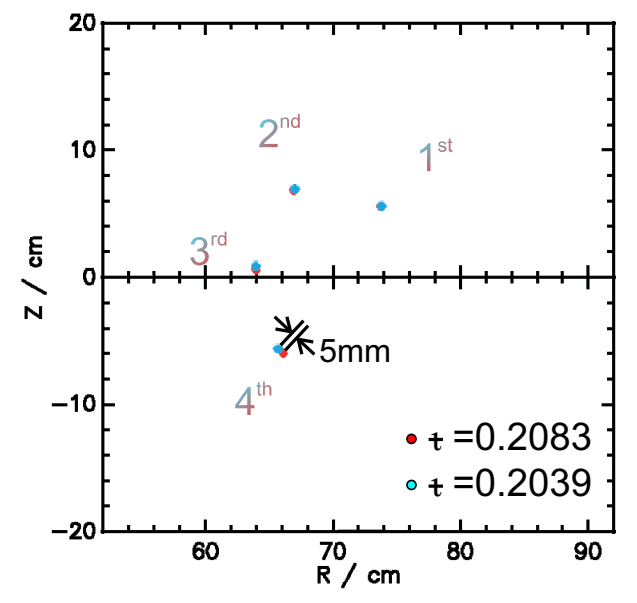

Figure 9: (Color online) Poincaré plot of the $1^{\text {st }}$ to $4^{\text {th }}$ intersection points in a poloidal plane of an electron drift path for two magnetic field configurations with $t=0.2083$ (red) and $t=0.2039$ (blue), calculated with the W7 code.

plot) for those two magnetic configurations. The value of $t$ for the second configuration was varied and the drift path of the electrons calculated, until the deviation for the $4^{\text {th }}$ transition was $5 \mathrm{~mm}$. Therefore, if two configurations can not be distinguished with this spatial accuracy, then their rotational transform can only differ by up to $\Delta t=0.004$.

Figures 10 a) and b) show a comparison between the measured points and the drift path of the respective electrons calculated with the W7 code. As can be seen the trajectories are not exactly the same but they are shifted and tilted. This produces a deviation up to $\Delta r=12 \mathrm{~mm}$ at the $4^{\text {th }}$ revolution, which can not be explained by the inaccuracy of the measurement even including the radial shift of the measured borders.

Two error sources could be identified but were not corrected during this proof of principle application. First, the conversion of measured coordinates in the stellarator system is sensitive to deviations of the actual mounted positions of the reference points. An inaccurate positioning causes all measured points to be shifted and tilted equally. Second, in some cases, a camera shift occurred due to strong magnetic forces during the measurement process. The reference points in the merged pictures were therefore misaligned and showed a displacement of up to $20 \mathrm{~mm}$. Whenever this error was obvious, the camera mount was tightened and the measurement was repeated, but a deformation of the optical system inside the camera could still have occurred. Depending on the set of two pictures used for the border measurement, this can produce shifts of data points in different directions for each set. 
a)

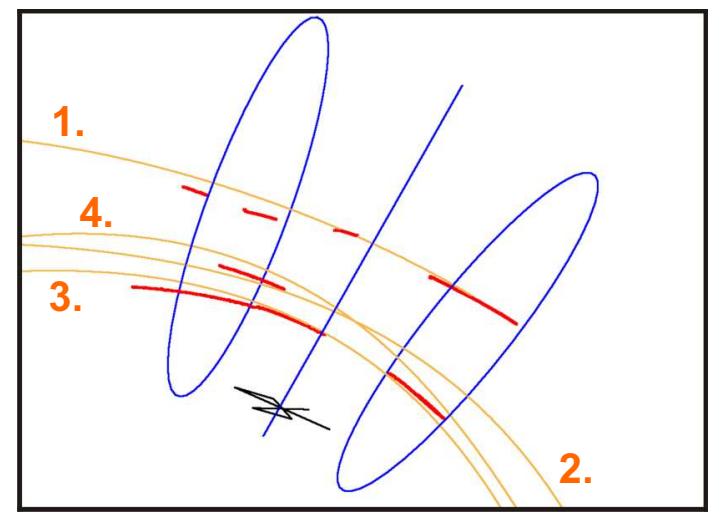

b)

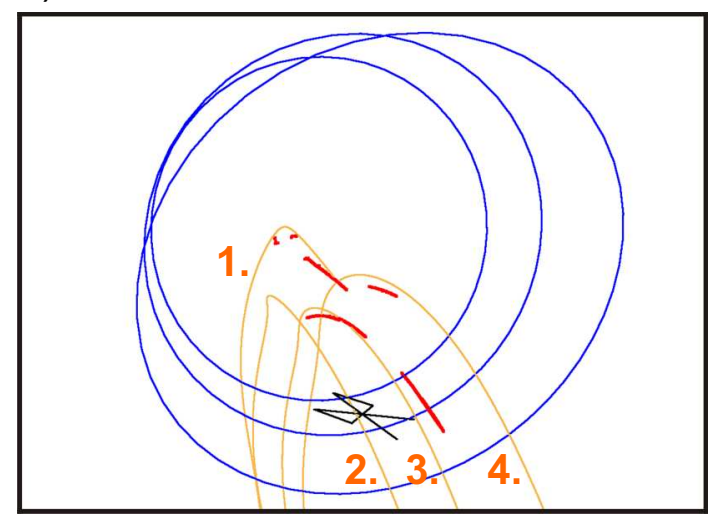

Figure 10: (Color online) Comparison of measured data (red) with numerically calculated drift trajectories (orange) in a topview (a) and an tangential view (b) on the plasma vessel contour (dark blue). The black figure represents the connected six reference points.

\section{OUTLOOK}

The proof of principle for this 3-dimensional measurement technique of magnetic field lines was given, although some work on improving the accuracy is still to be done. Especially the camera position determination by using a more accurate reference point-mount would be beneficial. In order to prevent an unintended interaction with the plasma in later fusion experiments, reference points compatible with plasma operation (heat-, vacuum- and radiation-resistant) should be utilized.

Nevertheless, this technique presents a complementary way of characterizing the vacuum magnetic field in a stellarator and can provide additional phase information on field error structures. It is currently under discussion to be used for the first tests of magnetic field configurations at the Wendelstein 7-X. Additional fields of application are the 
alignment and adjustment of toroidally separated plasma diagnostics aiming on certain parts of the magnetic field, like the magnetic axis or the last closed flux surface.

\section{Acknowledgments}

The authors wish to thank Dieter Aßmus, Stefanie Braun, Heinrich Laqua, Stefan Marsen, Norbert Paschkowski and Torsten Stange for the constant technical and theoretical support. Furthermore, they are grateful for the helpful discussions with Gerd Fußmann and the support of the institute of plasma physics of the Humboldt Universität zu Berlin.

[1] M. Otte and J. Lingertat, Proceedings 29th EPS Conference on Plasma Phys. and Contr. Fusion Montreux, 17-21 June 2002 ECA 26B, p 5.036 (2002).

[2] M. Hirsch, J. Baldzuhn, C. Beidler, R. Brakel, R. Burhenn, A. Dinklage, H. Ehmler, M. Endler, V. Erckmann, Y. Feng, J. Geiger, L. Giannone, G.Grieger, P. Grigull, H.-J. Hartfuß, D. Hartmann, R. Jaenicke, R. König, H. P. Laqua, H. Maaßberg, K. McCormick, F. Sardei, E. Speth, U. Stroth, F. Wagner, A. Weller, A. Werner, H. Wobig, S. Zoletnik, Plasma Phys. Control. Fusion 50 053001, pp 204 (2008).

[3] J. N. Talmadge, V. Sakaguchi, F. S. B. Anderson, A. F. Almagri, Physisc of Plasmas 8 Nr. 12, p. 5165 (2001)

[4] T. Y. Tou, B. D. Blackwell L. E. Sharp, Rev. Sci. Intrum. 62 (5), p. 1149

[5] O. Motojima, H. Yamada, A. Komori, N. Ohyabu, K. Kawahata, O. Kaneko, S. Masuzaki, A. Ejiri, M. Emoto, H. Funaba, M. Goto, K. Ida, H. Idei, S. Inagaki,N. Inoue, S. Kado, S. Kubo, R. Kumazawa, T. Minami, J. Miyazawa, T. Morisaki, S. Morita, S. Murakami, S. Muto, T. Mutoh, Y. Nagayama, Y. Nakamura, H. Nakanishi, K. Narihara, K. Nishimura, N. Noda, T. Kobuchi, S. Ohdachi, Y. Oka, M. Osakabe,T. Ozaki, B. J. Peterson, A. Sagara, S. Sakakibara, R. Sakamoto, H. Sasao, M. Sasao, K. Sato, M. Sato, T. Seki, T. Shimozuma, M. Shoji, H. Suzuki, Y. Takeiri, K. Tanaka, K. Toi, T. Tokuzawa, K. Tsumori, K. Tsuzuki, I. Yamada, S. Yamaguchi, M. Yokoyama, K. Y. Watanabe, T. Watari, Y. Hamada, K. Matsuoka, K. Murai, K. Ohkubo, I. Ohtake, M. Okamoto, S. Satoh, T. Satow, S. Sudo, S. Tanahashi, K. Yamazaki, M. Fujiwara, and A. Iiyoshi, Physisc of Plasmas 6 Nr. 5, p. 1843 (1999)

[6] M. Otte, R. Jaenicke, Stellarator News 100, p. 2-5 (2005).

[7] J. Wesson, Tokamaks, $2^{\text {nd }}$ edition, Clarendon Press-Oxford, pp. 40 (1997). 
[8] O. Neubauer, F. H. Bohn, A. Chudnovskij, B. Giesen, P. Hüttemann, M. Lochter, ANS Fusion Science and Technology 31, p. 154-158 (1997).

[9] Ralchenko, Yu., Kramida, A.E., Reader, J., and NIST ASD Team (2008). NIST Atomic Spectra Database (version 3.1.5), Online: http://physics.nist.gov/asd3 (June 30, 2009). National Institute of Standards and Technology, Gaithersburg, MD.

[10] I. Langmuir, Physical Review 2, Issue 6, pp. 450-486 (1913).

[11] M. Otte, D. Andruczyk, E. Holzhauer, J. Howard, R. König, L. Krupnik, H. P. Laqua, O. Lischtschenko, S. Marsen, J. Schacht, J. Urban, Y. Y. Podoba, J. Preinhalter, F. Wagner, G. B. Warr, A. Zhezhera, AIP Conf. Proc. 19. 993, p. 3-10 (2008).

[12] AICON 3D Studio, AICON 3D Systems GmbH, Online: http://www.aicon.de (June 2009). AICON 3D Systems GmbH, Braunschweig.

[13] C. Gourdon, Programme optimise de calculs numériques dans les configurations magnétique, Centre d'études nucléaires de Fontenay aux Roses, (1970). 